\title{
Establishment of Electronic Health Records in Developing Countries
}

\author{
Karan R. Shah \\ PVG's COET \\ Pune, India (MS)
}

\author{
Asif A. Tamboli \\ PVG's COET \\ Pune, India (MS)
}

\author{
Shubham S. \\ Pachpute \\ PVG's COET \\ Pune, India (MS)
}

\author{
Sudeep S. \\ Khare \\ PVG's COET \\ Pune, India (MS)
}

\author{
Sarang Saoji \\ PVG's COET \\ Pune, India (MS)
}

\begin{abstract}
The need of storing the personal health records was felt by the exponential increase in the risk and hazards that can be caused due to negligence and inadequacy in management of these health records especially in developing countries where these records are maintained manually. Electronic Health Records (EHR) plays a vital role in patient care and improving the quality of health services in a region. This paper focuses on the need and applications of the EHR which would help countries like India with a vast population to grow in an environment with good public health care services and management. Thus the structure of EHR would help to achieve an advanced and new medical application to suffice the requirements for a fast, reliable and better medical infrastructure.
\end{abstract}

\section{General Terms}

Networking, Database, Medical.

\section{Keywords}

Electronic Health Record, Database, Health Informatics.

\section{INTRODUCTION}

The Electronic Health Records (EHRs) are extensive electronic records of patient's health information keeping in mind the quality, safety and privacy of these sensitive personal records. Included in these records are patient demographics, progress reports, health issues, medications, vital signs, past medical history, immunizations and allergies. The EHR is an integration of multiple patient information systems. The EHR also includes the network that links these systems and databases which stores all these records. The most crucial component is the EHR database. The database should be structured, dynamic and consistent to achieve all the EHR objectives.

Storing health records in digital format helps to evade critical medical inaccuracies, and enhance health care facility. The proposed concept can be practically implemented by developing a pervasive and full-fledged networking application which will maintain and update the EHR dynamically.

\section{RELATED WORK}

In this section, this paper emphasizes on the essential aspects of implementation of EHR and its database structure.

\subsection{History}

For long time, the concept of electronic health has been discussed globally across all the countries, but it remains a work in progress everywhere. There are many reasons for the slow adoption of EHR ${ }^{[1]}$. Some include the scattered funding and administration of medical services, appropriate care to be taken while changing the existing models, lack of detailed research, and the sensitivity of the concept. There may also be concerns about the costs and complexities associated with e health implementation and the need to resolve issues about how it will affect practitioners and consumers alike. In a motive to implement EHR, the focus should be on concern about how e health will affect patient privacy.

E-health does indeed have great benefits and can be a boon in medical field, but harnessing these advantages require research and a careful procedure while negotiating a delicate balance between many benefits and concerns

\subsection{Australia}

The Australian PCEHR (Personally Controlled EHR) works on a local storage which is timely updated from the global storage. This is not a shared electronic health record system. Hospitals continue to maintain and use their own records as the primary data source while getting this local storage update on a time to time basis from the global storage. The PCEHR is thus a collection of documents and data uploaded from a variety of principal sources at different times ${ }^{[2]}$. But it will not always be clear if the information in local records is up to date. Integrating information from different sources may be time-consuming when there are multiple sources.

As expected, return of the PCEHR has been slow - in the first nine months, approximately 1, 00,000 consumers enrolled to use it. The complexity of the enrollment process has been a major limitation. Multiple models are being designed to improve consumer registration as it has been recognized that the existing systems are a barrier to uptake. Bringing all the information together into a single centralized medicines list is desirable but unlikely to be implemented in the short term. It will be important for all the diverse users to understand that a particular view of medicines information within the PCEHR may be incomplete ${ }^{[3]}$. Australia focused on achieving continuity in medication procedure even by implementing EHR. Thus outlining a partnership approach in which skill, knowledge and authority is shared among healthcare providers and consumers, for the end users benefit.

The development and implementation of national scale, shared electronic health records is difficult and expensive. It is difficult to find examples where such electronic systems have been delivered on time, on budget and have satisfied the needs of investors, government and end-users.

Australia has taken the initial steps towards a personal electronic health record. Some compelling challenges have already been evaded, but there are more ahead. The key to successful implementation of EHR will be a common understanding of the purpose and benefits of the new system detailed explanation of what the system can achieve now and 
in future should be developed and communicated to both medical practitioners and end-users ${ }^{[4]}$.

This is the start of an e-health evolution and progress will be incremental. It will be some time before benefits emerge. The PCEHR initiative will require long-term patience and commitment from the consumers, doctors and investors.

\subsection{Key Points From Related Work}

The progress of EHR implementation depends on intriguing consumers and doctors. Increasing the value is more likely to be achieved by undertaking ongoing assessment which results in rectification and adjustment of the system according to their needs.

\subsubsection{Data sharing}

There is potential for the EHR to offer new ways for medical practitioners and patients to share records effectively. Sharing and accessing of data could be more precisely. This would also be useful in emergencies and when patients are moving between healthcare settings, especially when there is a high risk of medication errors which can sometimes prove fatal.

\subsubsection{Consent}

The current opt-in consent model poses varied challenges for acquiring a huge mass of users to make the system beneficial. Settling trust in the security and accuracy of the data in the system will be important. At present there is little perceived benefit in the PCEHR for a patient's usual clinicians who already communicate and share information. Thus the detailed insight into benefits should be communicated properly.

\subsubsection{Avoiding problems}

Issues have been raised about the potential for risks and unintended consequences associated with the implementation of the EHR and the need for preventive measures to be taken. Incomplete and inaccurate data, such as missing some drugs should be overcome. Accuracy and currency of data will be a particular challenge for the PCEHR when there are various different sources of information. The challenge lies within proper integration of these records and to synchronize this information to build a complete record of the medicines that are being taken. In addition to this, as medical care involves more reliance upon electronically maintained information, the same robust processes of clinical control must be applied to it as done to all the other products used in the healthcare field. Safety governance for clinical information systems is long outstanding.

\section{PROBLEM STATEMENT}

The present working model of maintaining health records of a patient is manual and there exists certain issues while accessing these records dynamically to update the prescription details of a particular patient. Thus, there arises a need of proper and efficient model for maintaining these sensitive health records. This can be achieved by proper implementation and adoption of EHR providing stability to the conventional health record model.

\section{PROPOSED WORK}

The concept to implement EHR adoption in developed countries was felt from the need of the medicine prescription to be understood by the patient as well as records to be maintained in the form of electronic data.

The application is about automating the prescription process and maintaining patient's details along with prescription details through an Android application which will be connected to the XAMP server. EHR is a record in electronic format that is practically capable of being communicated across different health care institutions. In some cases this sharing can occur through a proper distributed network, local information systems and other global information exchanges.

A Doctor can store a patient's personal details and medical details at the time of patient's visit. All the details can be viewed and edited by the doctor. The data is backed up onto a remote server. Hence a doctor or a patient can access the respective data of patients from anywhere, at any time making it pervasive.

\subsection{Database Requirements}

The database is required both at the client side as well as the server side with different requirements. The client stores the local patient records while the server has to keep track of all the global records.

\subsubsection{Client Side: SQLite:}

SQLite is an open source sql database that implements a selfcontained transactional SQL engine without any dependence on the server. SQLite is currently found in many applications including several high-profile projects. Android comes with built in SQLite database implementation. It supports all the relational database features.

SQLite is an embedded SQL database engine. SQLite does not have a separate server process. SQLite stores data to a text file on a device. A complete SQL database with multiple tables, indices, triggers, and views, is contained in a single disk file. 


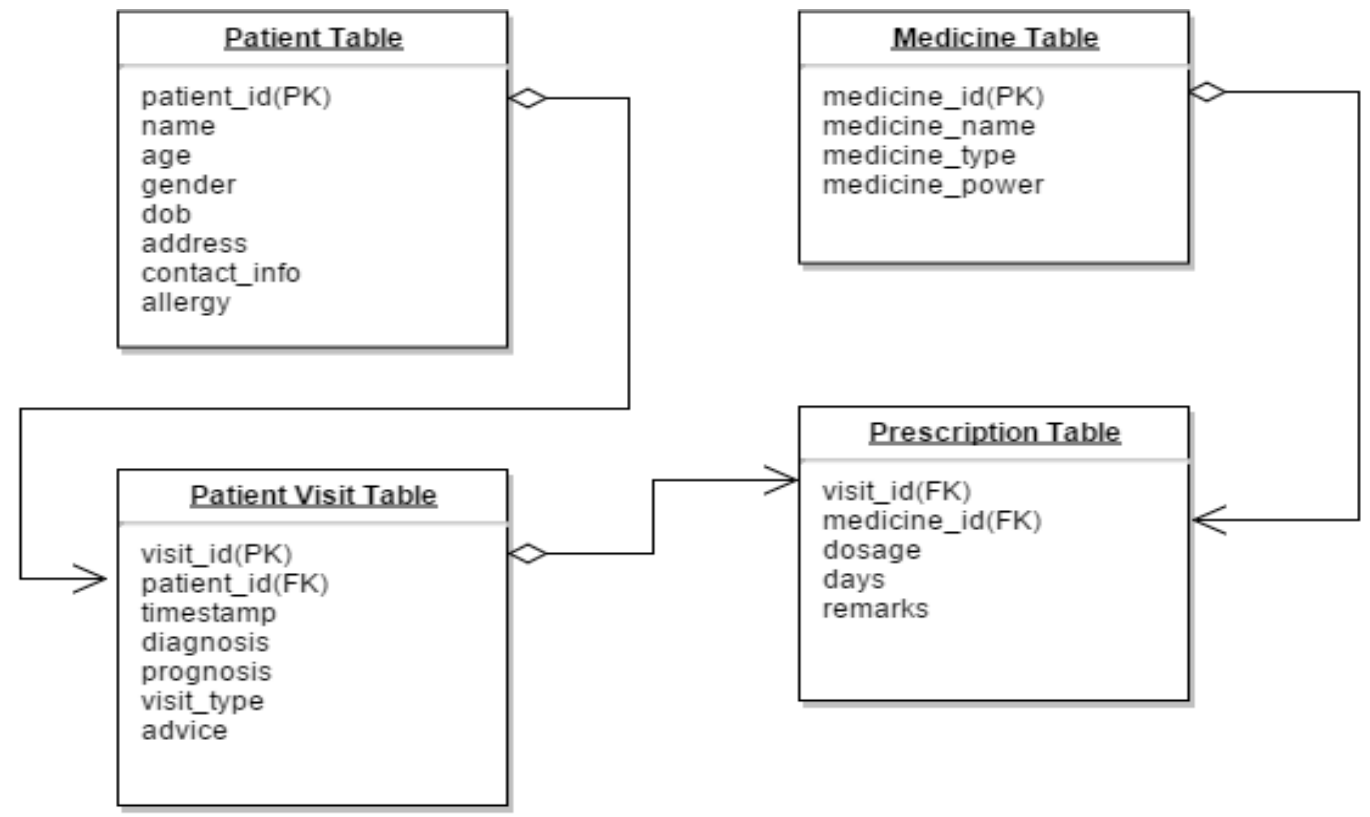

Fig. (1) Proposed Database Schema

\subsubsection{Server Side:}

MySQL:

MySQL is an open source relational database system. It is the database of the server side. A database is a collection of data. MySQL will be used on the server side to maintain the global EHR which is to be the combined version of various local EHRs. Thus, MySQL database makes itself useful for implementing full-fledged networking application for proper and efficient sharing of EHR among several health institutions.

MySQL is used on server side as it is a powerful database management system with much functionality. It can work on many operating systems and with diverse languages. It supports large databases while being customizable.

\subsection{Technical Specifications}

This section gives the details about all the technical details which will be used both at the client as well as server side.

\subsubsection{Client (Android)}

Android is a Linux-based operating system for mobile devices. The Android platform also allows end users to develop, install and use their own applications on top of the Android framework. The Android framework is licensed under the Apache License, with Android application developers holding the right to distribute their applications under their customized license.

The application to be developed will be an Android application because it has vast coverage amongst the crowd in the developing countries like India.

\subsubsection{Server}

Linux is one of the popular operating systems today. It is an open source operating system and free for use. Linux is not an user friendly system but one can make the most of it as per his needs which may not be found in proprietary or closed source operating systems. Linux being more secure and manageable according to any individual configurations, it could be easily used to meet all the necessary requirements of this project. It is also an operating system which could be seen as one of the fastest growing operating systems in the industry over the period with companies and top MNC's preferring Linux server/client operating systems over others. Viewing the entire scope of the project and predicting its future developments, Linux would be the best choice to use it as the server operating system supporting the various services provided though this idealogy.

Along with this operating system, a combination of PHP and MySQL technologies are used.

The application developed on the android platform for the client user interaction will be supported by this Linux operated server which will continuously service the requests generated y the application devices connected to it and store and backup the EHR database. Using Linux and other technologies with it reduces the cost of the entire proposed model as it is generally available under General Public License.

\subsection{Considerations Of End Users}

This section gives an insight to the considerations of all the end users be it medical practitioners, physicians or patients accessing their records.

\subsubsection{Quality}

EHR's may eventually help improve care coordination. Anyone using an EHR can view the patient's full medical and health details, that it result in elimination of predicting histories, and may be useful in improved care in emergency situations ${ }^{[5][6]}$. EHRs also improve prevention by providing doctors and patient's proper access to test results, considering patient information, and offering record-based suggestions for medical care ${ }^{[7]}$.

\subsubsection{Costs}

The high price involved in the implementation of EHR and the uncertainty with respect to the benefits from this implementation has a significant influence on EHR adoption [7]. The U.S. Authorities stated that the cost savings may be seen only in large institutions and not in small physician offices ${ }^{[8]}$. Doubts have been raised about cost saving from EHRs by many other researchers. 


\subsubsection{Time}

The implementation of EHR can result into decrease in recognition time of patients upon hospital admission. Many researchers have showed that since the adoption of EHR in developed countries, there has been relative decrease in time by approximately $67 \%$ has been recorded ${ }^{[9]}$.

\section{EXPERIMENTAL RESULTS:}

For experimental purpose, a dummy database of approximately 100 well known medicines was created. A basic android application was developed for doctors. For testing the same, it was provided to 5 local doctors who used it for prescribing medicines to a total of 50 patients. The feedback received can be displayed in the following chart:

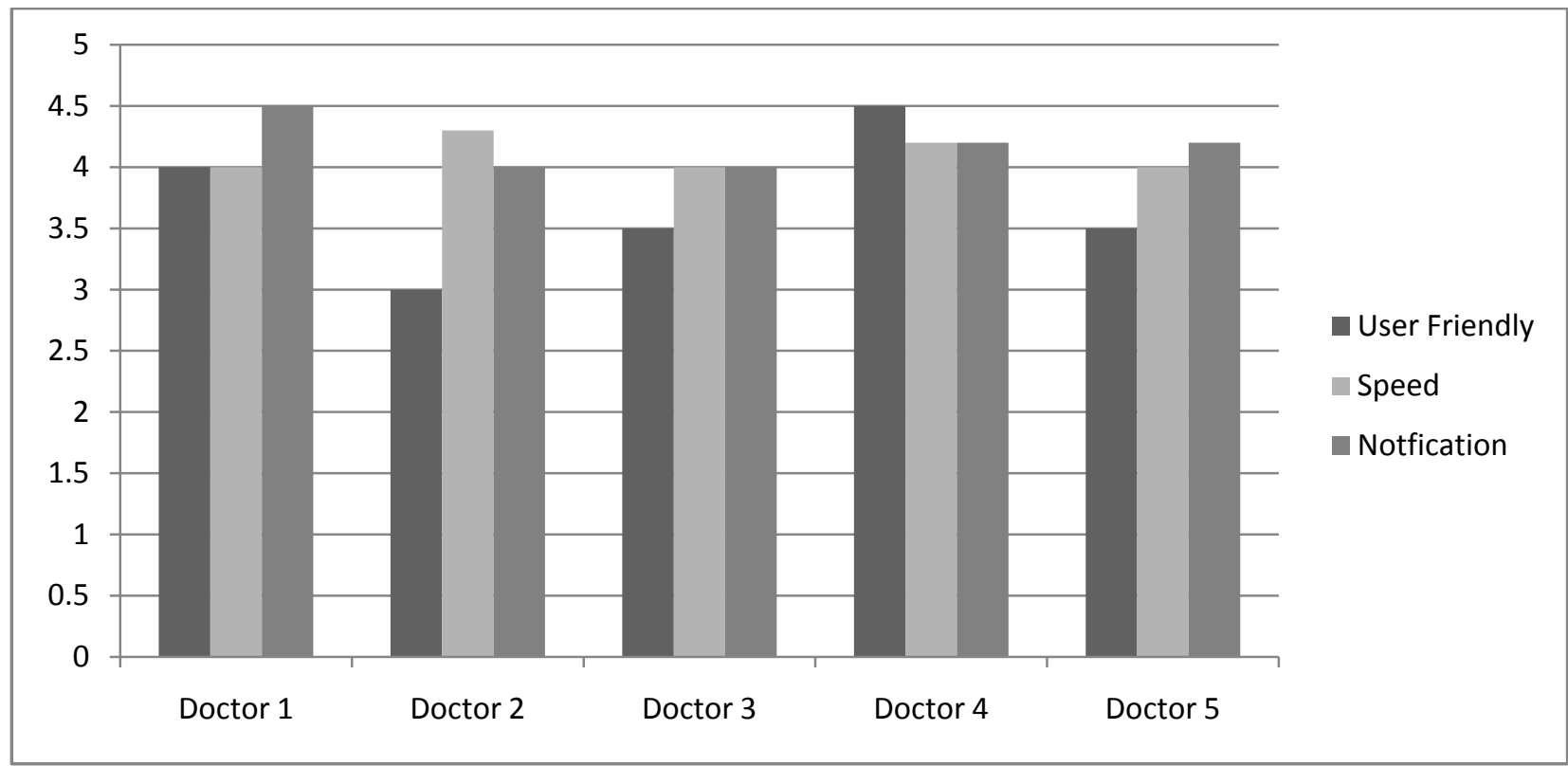

Fig. (2) Statistical analysis.

\section{ISSUES AND LIMITATIONS}

\subsection{Privacy and Security Issues}

The concept of a national centralized server model of healthcare data has been poorly received in the developed countries like USA, UK, and Germany. Privacy and security issues have always been a concern in the model of such a concept ${ }^{[9]}$.

Privacy concerns can be applied to paper as well as digital health records. Concerns such as 'data breach' will always be a possible issue where databases are involved. Records that are transferred over the Internet have to face the same security loopholes as any other type of data communication over the Internet ${ }^{[10]}$.

The privacy threat posed by the interoperability of a national network is a key concern. Accountability among all the parties that are involved in the processing of electronic transactions including the patient, physician office staff, and insurance companies, is the key to successful advancement of the EHR [10]

\subsection{Legal Issues}

Legal liability in all aspects of healthcare was an increasing problem in the 1990s and 2000s.

Failure or damages caused during installation or utilization of an EHR system has been feared as a threat in lawsuits. Similarly, it's important to recognize that the implementation of electronic health records carries with it significant legal risks ${ }^{[11]}$

This liability concern was more in small EHR systems. Larger EHR providers, government EHRs are able to withstand legal issues. While there is no argument that electronic documentation of patient visits and data brings improved patient care, there is increasing concern that such documentation could open physicians to an increased incidence of malpractice suits ${ }^{[12]}$.

\section{CONCLUSION}

In this paper, a pervasive, medical and an efficient networked data model for the EHR database using relational database have been proposed. Because patient problems are the most important driver for medical queries in healthcare, this model describes a problem oriented record base. This logical design did not present data attributes for entities to make it simple and to make the design as generic as possible. The proposed model allows dynamic additions to the EHR database applied smoothly and with minimum hindrance. The dynamic, social, and medical aspects of the model enhance the prescription process using the EHR database which also helps to enhance the healthcare situations of medical institutions. The next step will be to implement this data model and develop the proposed application. Moreover, this would build a medical prescription support system which collects patient data from EHR and takes decisions to support healthcare personnel to make accurate medical prescriptions. This proposed concept will thus act as a catalyst in future adoption of EHR standards in all the developing countries which will prove to be a big boon in medical field.

\section{REFERENCES}

[1] Arnold, Steven L.; Klein, Gary M. (2008). "Electronic Health Records: A Global Perspective". Healthcare Information and Management Systems Society (HIMSS).

[2] McDonald, Kate. (2013). "Half a million PCEHR registrations still achievable". Pulse+IT Magazine. 
[3] Cebul, Randall D.; Love, Thomas E.; Jain, Anil K.; Hebert, Christopher J. (2011). "Electronic Health Records and Quality of Diabetes Care". New England Journal of Medicine.

[4] Mason, Moya K. (2005). "What Can We Learn from the Rest of the World? A Look at International Electronic Health Record Best Practices".

[5] Greenhalgh, Trisha; Potts, Henry W.W.; Wong, Geoff; Bark, Pippa; Swinglehurst, Deborah (2009). "Tensions and Paradoxes in Electronic Patient Record Research: A Systematic Literature Review Using the Meta-narrative Method". Milbank Quarterly.

[6] Gabriel, Barbara (2008). "Do EHRs Make You a Better Doctor?" Physicians Practice.

[7] Himmelstein, David U.; Wright, Adam; Woolhandler, Steffie (2010). "Hospital Computing and the Costs and Quality of Care: A National Study". The American Journal of Medicine.
[8] Shah, Shahid. (2009) "Column: Why MDs Dread EHRs". Journal of Surgical Radiology.

[9] Chaudhry, Basit; Wang, Jerome; Wu, Shinyi; Maglione, Margaret; Mojica, Walter; Roth, Elizabeth; Morton, Sally C.; Shekelle, Paul G. (2006). "Systematic Review: Impact of Health Information Technology on Quality, Efficiency, and Costs of Medical Care".

[10] Wager, K.; Lee, F.; Glaser, J. (2009). Health Care Information Systems: A Practical Approach for EHR Management".

[11] Schwartz, Shelly K. (2012) "Can Technology Get You Sued?"

[12] Dunlop, Laura. (2007). "Electronic Health Records: Interoperability Challenges and Patient's Right for Privacy". Shidler Journal of Computer and Technology. 